Case Report

\title{
Iatrogenic esophageal perforation with mediastinitis, multidisciplinary management: a case report
}

\author{
Juliana Mancera ${ }^{1}$, Anibal M. Ariza ${ }^{2}$, Mauricio Pelaez ${ }^{2}$, Sebastian Benavides ${ }^{2}$, \\ Alfonso C. Marquez ${ }^{2}$,
}

${ }^{1}$ Department of Medicine, Pontificia Universidad Javeriana, Bogota, Colombia
${ }^{2}$ Department of Surgery, Hospital Universitario San Ignacio, Bogota, Colombia

Received: 09 September 2021

Accepted: 04 October 2021

\section{*Correspondence:}

Dr. Juliana Mancera,

E-mail: mancera.j@javeriana.edu.co

Copyright: (C) the author(s), publisher and licensee Medip Academy. This is an open-access article distributed under the terms of the Creative Commons Attribution Non-Commercial License, which permits unrestricted non-commercial use, distribution, and reproduction in any medium, provided the original work is properly cited.

\begin{abstract}
Acute mediastinitis is a low incidence pathology, but it is associated with a high mortality rate. Iatrogenic lesions are the most common cause of mediastinitis secondary to esophageal perforation. Early diagnosis and surgical treatment are the most important factors in the treatment of patients with this condition. 83-year-old female patient, with a history of left saphenectomy due to venous insufficiency with difficult intubation two days prior to the emergency consultation. She was admitted to the emergency room due to dyspnea, hemoptysis, chest pain, and right hemifacial edema. A chest tomography was performed with findings suggestive of esophageal perforation or airway injury. Later, in an upper digestive tract study, extravasation of the contrast medium was documented in the right posterolateral wall of the cervical esophagus with a collection in the middle mediastinum. The patient was taken for drainage of mediastinitis by right thoracoscopy and the presence of a perforation in the cervical esophagus was confirmed with an intraoperative endoscopy. Esophageal repair was performed, with drainage of the prevertebral space and the superior mediastinum by a left longitudinal cervicotomy. Postoperatively, she received antibiotic and enteral nutritional support by a nasojejunal tube. Low output fistula of the cervical esophagus, organized by a drain, was documented, which closed after 4 weeks of conservative management. Iatrogenic esophageal perforation with mediastinitis is a very rare entity with a high mortality. Early surgical treatment is the most important prognostic factor in patients with mediastinitis due to esophageal perforation.
\end{abstract}

Keywords: Esophagus, Mediastinitis, Esophageal perforation, Thoracoscopy, Esophageal fistula

\section{INTRODUCTION}

Acute mediastinitis is the infection or inflammation of the loose connective tissue that surrounds the mediastinum. Despite its low incidence, it is associated with a high mortality rate. ${ }^{1}$ Among the most frequent causes are esophageal perforation, infectious processes (abscesses) at the level of the neck or of odontogenic origin, and infection secondary to cardiovascular surgery due to sternotomy. ${ }^{2,3}$ In particular, iatrogenic lesions are the most common cause of mediastinitis secondary to esophageal perforation. The most frequent clinical manifestations are retrosternal pain, dysphagia, and fever and in some cases there may be deterioration of the respiratory status. ${ }^{1,4,5}$ Contrast computed axial tomography is the imaging modality of choice for the diagnosis and follow-up of this pathology. Imaging findings that suggest the presence of esophageal perforation with mediastinitis are: fluid collection in the mediastinal space, extraluminal gas, pleural effusion, attenuation of mediastinal fat, mediastinal widening, thickening of the esophageal walls, and contrast medium extravasation. ${ }^{6}$ The esophagram (study of the upper digestive tract) with oral ingestion of water-soluble contrast can provide the precise location of the perforation to define the surgical approach; endoscopic 
exploration is not recommended except for the extraction of foreign bodies. ${ }^{1}$

The treatment of choice for the management of mediastinitis due to esophageal perforation is surgical drainage and debridement of the necrotic tissue, various surgical approaches have been described depending on the location of the perforation, either by cervicotomy, thoracotomy or thoracoscopy. ${ }^{7-10}$ In addition to surgical treatment, it is essential to achieve adequate control of the septic focus with early initiation of broad-spectrum antibiotic therapy, which can later be narrowed according to the microorganism obtained in the drainage cultures. $^{1,11}$ Additionally, the patient should remain nothing by mouth, so it is indicated to start enteral or parenteral nutritional support early. ${ }^{1,4,11}$

\section{CASE REPORT}

83-year-old female patient with a history of left saphenectomy due to venous insufficiency with difficult intubation two days prior to emergency consultation for dyspnea, hemoptysis, chest pain radiating to the dorsal region and right hemifacial edema. Upon admission in poor general conditions, heart rate 120 beats per minute, respiratory rate 25 per minute, oxygen saturation down to $82 \%$ on room air with supplemental oxygen requirement per ventury at $40 \%$ to achieve a saturation of $90 \%$. On physical examination, presented subcutaneous emphysema that extended throughout the chest wall to the fifth costal arch, and pulmonary auscultation with the presence of bilateral rhonchi. Chest X-ray showed right pleural effusion, bilateral reticular infiltrates, and subcutaneous emphysema (Figure 1).

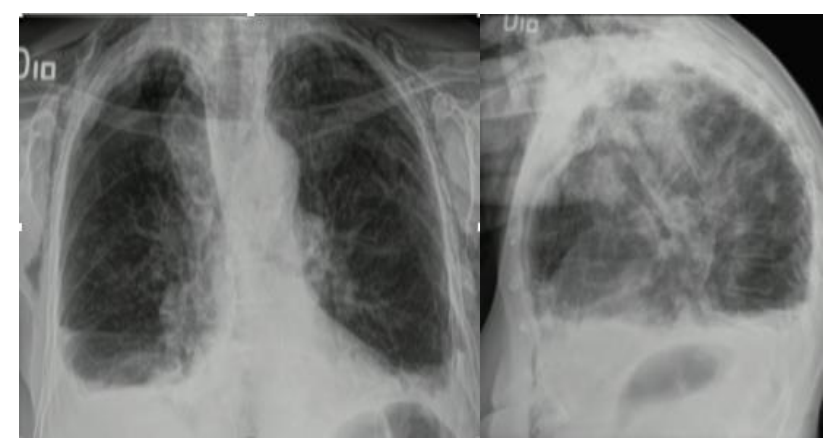

Figure 1: Chest X-ray, right pleural effusion and bilateral reticular infiltrates.

Chest tomography studies were expanded with findings of bilateral pneumothorax, pneumomediastinum, pulmonary embolism with thrombi in segmental branches for the lower lobe and lingula, bilateral pleural effusion and peri-esophageal gas (Figure 2), for which esophageal perforation versus lesion of the airway was suspected. A bilateral closed thoracostomy was performed with a right hydropneumothorax and a left pneumothorax. Studies with upper digestive tract were complemented with evidence of contrast medium extravasation in the right posterolateral wall of the proximal esophagus with an associated collection in the middle mediastinum (Figure 3).

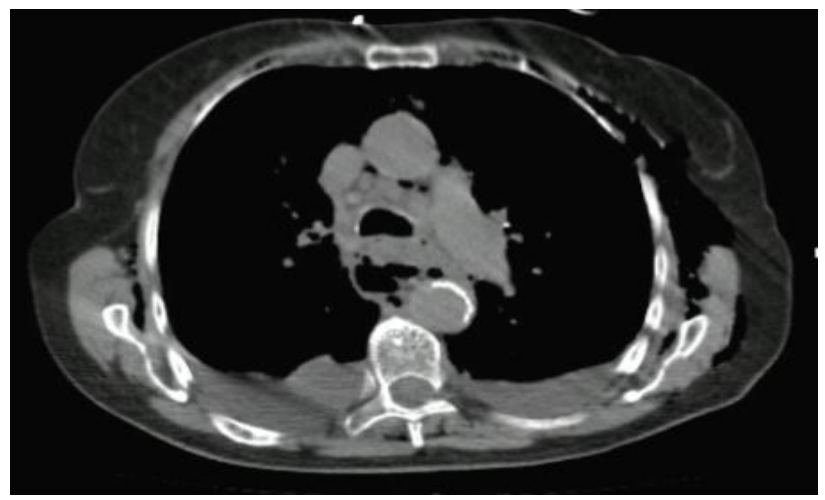

Figure 2: Chest CT Angiogram. Bilateral pneumothorax and pneumomediastinum, pulmonary embolism with thrombi in segmental branches for the lower lobe and lingula, bilateral pleural effusion, perioesophageal gas, no trachea and airway continuity solution.

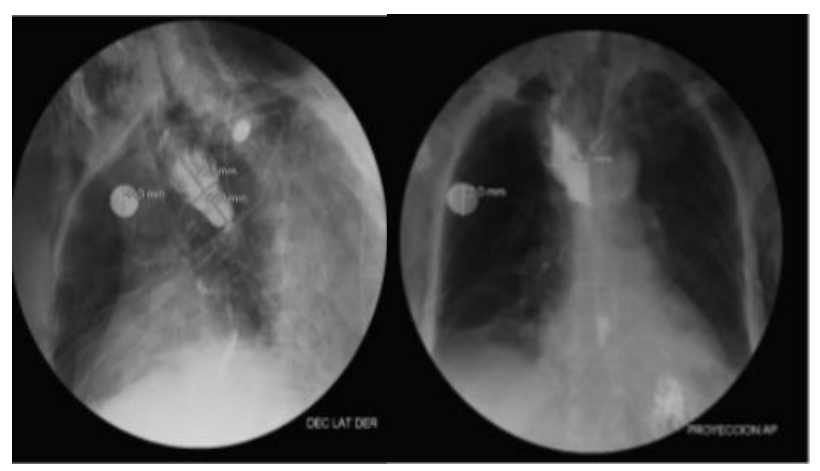

Figure 3: X-ray of the digestive tract: fistulous path dependent on the right posterolateral wall of the proximal esophagus at the level of the vertebral body of $\mathbf{T} 2$, it ends in a space in the middle mediastinum whose dimensions are $76 \times 27 \mathrm{~mm}(\mathrm{~L} \times \mathrm{AP} \times \mathrm{T})$ and presents stasis of the contrast medium without it being subsequently evacuated.

The patient was taken to the operating room for right thoracoscopy where drainage of the mediastinitis was performed without evidence of perforation of the thoracic esophagus (Figure 4). An intraoperative upper digestive tract endoscopy was performed where the presence of a 6 $\mathrm{mm}$ perforation was confirmed in the cervical esophagus at the level of the cricopharyngeal (Figure 5). Subsequently, a left longitudinal cervicotomy was performed with drainage of the cervical pre-vertebral space and the superior mediastinum, and subsequent primary esophageal repair with suture. During surgery, a culture of the pleural membranes was taken, with a report that documented Staphylococcus aureus resistant to oxacillin, so antibiotic coverage was oriented and management with ampicillin sulbactam plus vancomycin was indicated for 4 weeks. Diet was restricted to nothing 
by mouth and enteral nutrition was provided by a nasojejunal tube.

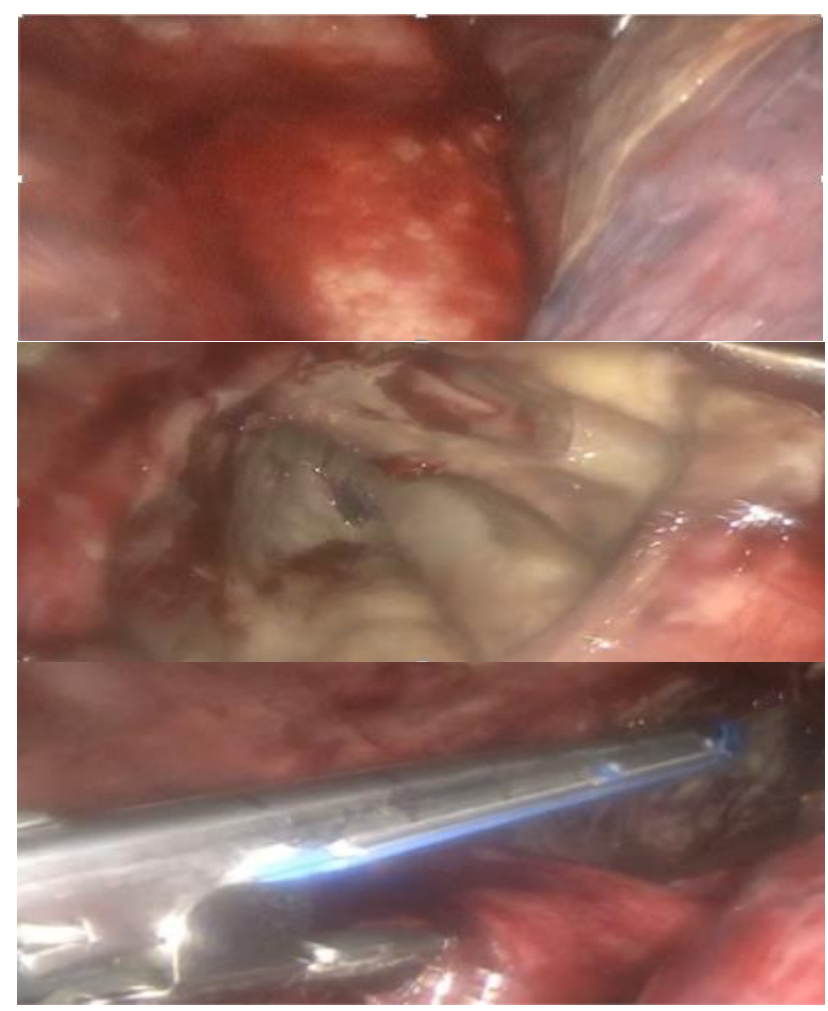

Figure 4: Right thoracoscopy: mediastinitis without documenting perforation of the thoracic esophagus.

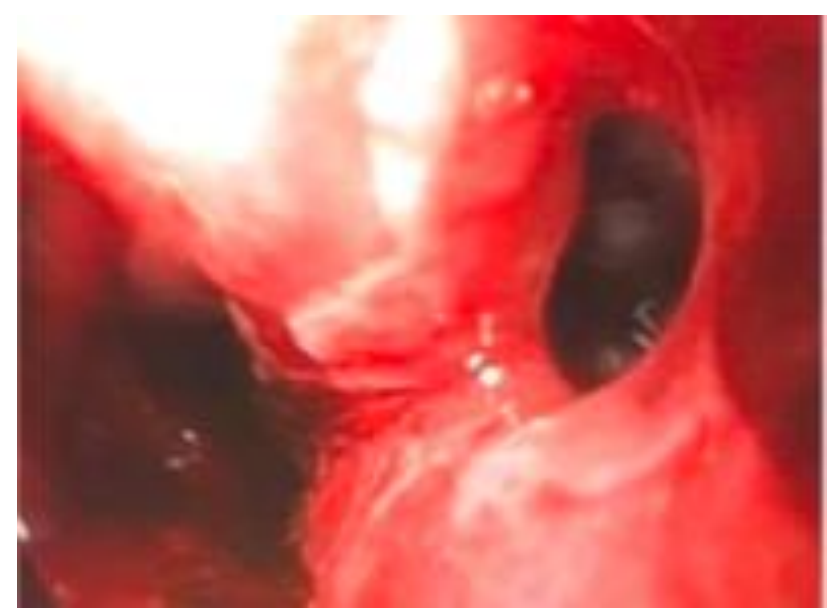

Figure 5: Upper digestive tract endoscopy: $6 \mathrm{~mm}$ perforation in the cervical esophagus at the level of the cricopharyngeal muscle.

Postoperatively, the patient presents an adequate clinical course with minimal complications, and complete resolution of the signs of systemic inflammatory response. On the seventh day, presented a cervical fistula, organized around a drain positioned during the surgical procedure. Spontaneous closure of the fistula was achieved after 4 weeks with conservative management. In the follow-up she was asymptomatic.

\section{DISCUSSION}

Acute mediastinitis is defined as the inflammation or infection of the connective tissue that surrounds the structures that make up the mediastinum, which despite its low incidence has a high mortality, being considered the most lethal form of infection in the chest. ${ }^{1,4}$ This entity has an approximate mortality of 18 to $44 \%$, which can increase even up to $67 \%$ in patients with comorbidities, so that in most of these cases the patients will require admission and management in the intensive care unit. $^{12,14}$

Among the main causes of mediastinitis are esophageal perforation, infectious processes at the neck level or of odontogenic origin that configure a descending mediastinitis, postoperative mediastinitis secondary to an infection after esophageal and cardiac surgery, orotracheal intubation and endoscopic procedures. Specifically in the case of esophageal perforation mediastinitis, iatrogenic lesions correspond to approximately $70 \%$, while $15 \%$ are due to spontaneous esophageal rupture. $^{2-4,12}$

The signs and symptoms that occur in mediastinitis are not very specific and since there are multiple etiologies, they will depend on the underlying cause. In most cases, regardless of the cause, patients present with fever, chills and tachycardia, specifically in the case of iatrogenic esophageal perforation mediastinitis, patients additionally present retrosternal pain, dysphagia and in some cases deterioration of the respiratory pattern. ${ }^{1,4,5,14}$

The computed tomography of the neck and chest is the image of choice for the diagnosis of mediastinitis with a sensitivity and specificity of $100 \%$, among the imaging findings are: mediastinal fluid collection, extraluminal gas, pleural effusion, fat attenuation mediastinal, mediastinal widening, esophageal thickening and extravasation of the contrast medium. ${ }^{4,6,14}$ Among other studies, an upper digestive tract X-ray with oral ingestion of water-soluble contrast is recommended, since it has the utility of providing the precise location of the esophageal perforation, allowing the surgical approach to be adequately planned. ${ }^{1}$

Regarding management, the mainstay of treatment is surgical drainage, which has a direct impact on mortality when it is established early and is associated with empirical antibiotic coverage. The objective of surgical management is to perform a cleaning of the affected spaces added to a debridement of the necrotic tissues, in turn allowing the taking of cultures to direct the antibiotic coverage. As the only exception, surgical management is not performed in the case of minimal perforations in the cervical esophagus with axial computed tomography that confirms the absence of contrast medium into the mediastinum, in the rest of the cases it should always be done. $^{4,13}$ 
In the case of cervical esophageal perforation, the approach is made through a left cervicotomy along the lower third of the sternocleidomastoid muscle (SCM), which is retracted laterally with the carotid sheath, the middle thyroid vein and the muscle are divided. Omohyoid, trachea and esophagus retract medially. A dissection of the esophagus is performed in the posterior part along the retropharyngeal plane, later the devitalized tissue is debrided with blunt dissection in the mediastinum, posterior to the esophagus and anterior to the prevertebral fascia, and finally an esophageal repair is performed only if the perforation site is clearly visible. This is carried out through an incision in the muscle layer longitudinally along the muscle fibers superior and inferior to the perforation, achieving an exposure of the mucosa in the entire extension of the lesion, finally a suture is made in two planes starting with the mucosa and later the muscular layer, leaving a drain in the site adjacent to the lesion. ${ }^{13}$

In the case of our patient, we consider that the history of difficult intubation performed two days prior to the emergency department visit was the cause of the perforation of the cervical esophagus, since it has a clinical presentation similar to that previously described. In this case, the diagnostic suspicion that led to the performance of the computed tomography and the X-ray of the upper digestive tract was essential, which allowed locating the perforation, initially with suspicion of locating it in the thoracic esophagus. For this reason, it was decided to perform a thoracoscopic approach; however, since only findings of mediastinitis were documented but thoracic esophageal perforation was ruled out, it was decided to use an intraoperative esophagogastroduodenoscopy that allowed locating the perforation at the cervical level, requiring an approach by left cervicotomy to complete the washing and debridement of the necrotic tissue, and additionally allowing to perform the esophageal repair at the level of the perforation.

This case highlights the importance of early diagnosis, medical treatment with targeted antibiotic therapy, enteral nutrition, and surgical intervention for drainage and repair, in the management of patients with acute mediastinitis secondary to esophageal perforation. A multidisciplinary approach, in this case from group made up of general surgery, thoracic surgery and gastroenterology, resulted in an early diagnosis and effective treatment, leading to a successful outcome for the patient, who had an uncomplicated postoperative course.

\section{CONCLUSION}

Mediastinitis secondary to esophageal perforation is a rare condition but with high mortality. It is a critical condition, in which timely surgical treatment is the mainstay of management along with supportive measures and targeted antibiotic coverage. The preoperative study is of vital importance to make a proper diagnosis and guide the surgical approach. Multimodal management with a multidisciplinary team leads to better results and outcomes for the patient.

\section{Funding: No funding sources \\ Conflict of interest: None declared \\ Ethical approval: Not required}

\section{REFERENCES}

1. Zelada I, Farina C, Lee C, Agüero Á. Acute Mediastinitis. Retrospective Analysis of 12 Cases. Cir Paraguaya. 2019;43(1):12-5.

2. Kiernan PD, Hernandez A, Byrne WD, Bloom R, Dicicco B, Hetrick V, et al. Descending cervical mediastinitis. Ann Thorac Surg. 1998;65(5):1483-8.

3. Dajer-Fadel WL, Ibarra-Pérez C, SánchezVelázquez LD, Borrego-Borrego R, NavarroReynoso FP, Argüero-Sánchez R. Descending necrotizing mediastinitis below the tracheal carina. Asian Cardiovasc Thorac Ann. 2014;22(2):176-82.

4. Martínez Vallina P, Espinosa Jiménez D, Pérez LH, Triviño Ramírez A. Mediastinitis. Arch Bronconeumol. 2011;47(8):32-6.

5. Misiak P, Jabłoński S, Piskorz Ł, Dorozała L, Terlecki A, Wcisło S. Oesophageal perforation Therapeutic and diagnostics challenge. Retrospective, single-centre case report analysis (2009-2015). Pol Prz Chir Polish J Surg. 2017;89(4):1-5.

6. Giménez A, Franquet T, Erasmus JJ, Martínez S, Estrada P. Thoracic complications of esophageal disorders. Radiographics. 2002;22.

7. Ohri SK, Liakakos TA, Pathi V, Townsend ER, Fountain SW. Primary repair of iatrogenic thoracic esophageal perforation and Boerhaave's syndrome. Ann Thorac Surg. 1993;55(3):603-6.

8. Burnett CM, Rosemurgy AS, Pfeiffer EA. Lifethreatening acute posterior mediastinitis due to esophageal perforation. Ann Thorac Surg. 1990;49(6):979-83.

9. Seal KA, Kini S. Esophageal perforation and mediastinitis. Pract Emerg Resusc Crit Care. 2013;278-83.

10. Brinster CJ, Singhal S, Lee L, Marshall MB, Kaiser LR, Kucharczuk JC. Evolving options in the management of esophageal perforation. Ann Thorac Surg. 2004;77(4):1475-83.

11. Cross MR, Greenwald MF, Dahhan A, Esposito S. Esophageal perforation and acute bacterial mediastinitis: Other causes of Chest Pain That can be easily missed. Med (United States). 2015;94(32).

12. Jabłoński S, Brocki M, Kordiak J, Misiak P, Terlecki A, Kozakiewicz M. Acute mediastinitis: evaluation of clinical risk factors for death in surgically treated patients. ANZ J Surg. 2013;83(9):657-63.

13. Cho JS, Kim YD, Hoseok I, Lee SK, Jeong YJ. Treatment of mediastinitis using video-assisted 
thoracoscopic surgery. Jeong $\mathrm{Su}$ Cho.Eur J Cardiothorac Sur. 2008;34(3):520-4.

14. Pastene B, Cassir N, Tankel J, Einav S, Fournier PE, Thomas P, et al. Mediastinitis in the intensive care unit patient: a narrative review. Clin Microbiol Inf. 2020;26(1):26-34.
Cite this article as: Mancera $\mathrm{J}$, Ariza AM, Pelaez M, Benavides S, Marquez AC. Iatrogenic esophageal perforation with mediastinitis, multidisciplinary management: a case report. Int Surg J 2021;8:3444-8. 